\title{
Experimental Study of the Distribution of Temperature Fields in the Butt Contour of a Welded Joint Made of Carbon and High-Alloy Structural Steels
}

\author{
Lubov Mironova ${ }^{1, *}$, Ruclan Nigay $^{2}$, and Evgenij Nigay $^{2}$ \\ ${ }^{1}$ Moscow Aviation Institute (State National Research University), Moscow, Russia \\ ${ }^{2}$ Russian University of Transport (MIIT), Russia
}

\begin{abstract}
A graphic-analytical method for determining temperature fields in the butt contour of a welded joint of a thin-walled tube with a base is presented. The method is based on the experimental data of temperature measurement at the reference points of the structure. The features of the elements of the welded joint made of carbon and high-alloy structural steels and the modes of electric arc welding were taken into account. A graphic-analytical method for determining temperature fields is proposed, which, on the basis of the theory of welding strains and stresses, makes it possible to determine temperature stresses and strains, both in the elements of the joint and in the contour of the weld.
\end{abstract}

\section{Introduction}

The creation of a welded joint is based on the melting of the auxiliary and base metal in the welding bath, provided by the arc gorenje with subsequent solidification of the weld [1]. In this case, there is a heat transfer and heat exchange with the environment. Finding temperature fields by methods of thermal conductivity and heat transfer is not acceptable in many cases, since it is not always possible to take into account the heating conditions that are carried out in welding processes by a moving highly concentrated heat source [2].

Also, a wide variety of forms of welded joints, grades of welded materials and welding methods do not allow us to apply any unified approaches and methods to determine the levels of temperature stresses arising under the influence of high temperatures localized in a narrow zone of the weld [3]. Therefore, the study of thermal processes and the thermally stressed state of welded joints remains an urgent task in ensuring the strength and reliability of machine-building products. Special attention is paid to experimental methods for determining temperature fields in the technological cycle of welding of a welded joint.

This paper presents the results of an experimental study of the distribution of temperature fields in the butt circuit of a welded joint made of carbon and high-alloy structural steels. As a sample, the "shell - plate" welded joint was used, which is widely used in the construction of power apparatuses.

\footnotetext{
*Corresponding author: mironova_lub@mail.ru
} 


\subsection{Analysis of the welded structure}

Fig. 1. shows the welded joint and the scheme of applying the weld. A welded joint from the point of view of mechanics can be conditionally considered as a "shell - plate" joint. A pipe of considerable length is welded to the base of sufficient thickness. The material of the plate is low-carbon steel 20, the material of the shell (tube) is high-alloy steel of the austenitic class. The technological properties of these steels in terms of weldability are good.
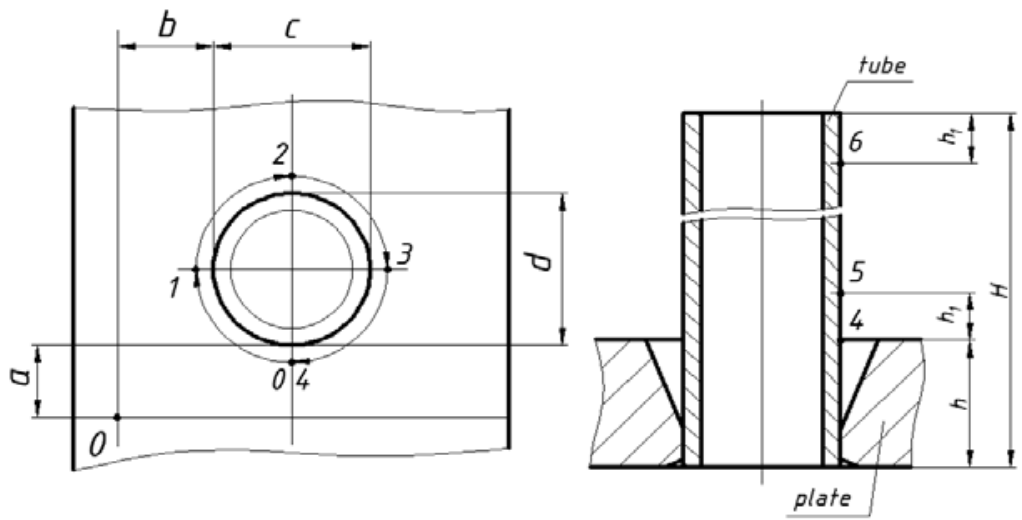

Fig. 1. Sample and scheme of welding seam: 1 is base; 2 is tube.

The peculiarity of the studied design is the different thickness of the elements in the contour of the welded joint, which in the conditions of thermal loading by a mobile heat source can lead to a significant warping of the structure.

\subsection{Features of the technology of creating a welded joint}

The welded joint is non-rotating. The welding method is manual electric arc welding by melting the electrode. The following factors are attributed to the peculiarities of the welding process. Multi-pass welding with overclocking of the weld is carried out. Seams are made manually with the breakdown of the contour of the joint into several sections. The uneven movement of the point heat source along the connection contour leads to an uneven thermal stress state of the near-seam zone and its deformation. During the application of the rollers during the welding of the shell elements, the welding modes were changed, i.e. welding was carried out on "slow" and "fast currents". At the reference points of the weld contour in the direction of the arc movement after each welding pass, the temperature was measured with a contact thermometer TK-5 at certain time intervals.

\section{The essence of the experiment. Methodology and results}

Studies of the thermal and deformation processes of the selected sample were carried out under different conditions of thermal loading on the welding current: with a decrease and increase in the welding current by $6 \%$. within the normal mode. Five welding passes were performed, the welding current varied from $150 \div 170 \mathrm{~A}$.

After welding each section of the contour joint (0-1; 1-2; 2-3; 3-4, Fig. 1), temperature measurements were carried out, both in the contour itself and along the tube height (at points $4 ; 5 ; 6$, Fig. 1) 
Temperature measurements were carried out after the roller was applied in accordance with the developed method. The welded joint was cooled in natural conditions in air at a temperature of $20^{\circ} \mathrm{C}$.

The results of the study of thermal processes and the distribution of the temperature field along the length of the pipe are presented in [4].

\subsection{Results}

The results of the temperature measurement in the contour connection are shown in Table 1.

Table 1. Temperature change in the contour connection after the fifth pass

\begin{tabular}{|c|c|c|c|c|c|c|c|}
\hline \multicolumn{2}{|c|}{ Time, c } & \multirow{2}{*}{ Temperature, ${ }^{\circ} \mathrm{C}$} & \multicolumn{5}{|c|}{ Characteristic points } \\
\hline$t_{i}$ & $\Delta t$ & & 0 & 1 & 2 & 3 & 4 \\
\hline 120 & 120 & $T_{1}$ & 200 & 250 & 245 & 270 & 303 \\
\hline 420 & 300 & $T_{2}$ & 250 & 225 & 245 & 210 & 250 \\
\hline \multicolumn{3}{|c|}{$\Delta T,{ }^{\circ} \mathrm{C}$} & +50 & -25 & 0 & -60 & -53 \\
\hline \multicolumn{3}{|c|}{$\begin{array}{l}\text { Rate of temperature change }|\Delta T| / \Delta t \text {, } \\
{ }^{\circ} \mathrm{C} / \mathrm{sec}\end{array}$} & 0.166 & 0.083 & 0 & 0.2 & 0.176 \\
\hline \multicolumn{3}{|c|}{$\begin{array}{l}\text { Average value of the rate of } \\
\text { temperature change, }{ }^{\circ} \mathrm{C} / \mathrm{sec}\end{array}$} & \multicolumn{5}{|c|}{0.125} \\
\hline
\end{tabular}

The rate of temperature change was determined by the formula

$$
\frac{\Delta T}{\Delta t}=\frac{\left|T_{2}-T_{1}\right|}{t_{2}-t_{1}}
$$

Here $T_{1}$ and $T_{2}$ are the temperature at the first and second measurements, $t_{2}$ and $t_{1}$ are the intermediate values of the temperature measurement time: $t_{1}=120 \mathrm{~s}, t_{2}=420 \mathrm{~s}$. The time interval during which the rate of cooling of the welded joint in air was determined was $\Delta t=$ $300 \mathrm{~s}$.

\section{Study of temperature fields based on the results of the experiment}

It is known that the method of electric arc welding is based on local heating of the metal before melting by an electric arc. At the same time, the heating source is characterized by a high concentration of energy and a relatively short length. Therefore, often proposed methods for studying temperature fields based on the theory of thermal conductivity are presented in relation to such specific heat sources, taking into account standard models in the form of a semi-infinite body, plate or rod. At the same time, it is believed that in the high temperature region, the temperature field is largely influenced by the features of heat input and, to a lesser extent, by the boundary conditions [5]. Here it is necessary to consider the features of welding sources in terms of heat distribution, action time, and movement relative to the heated body.

The proposed solutions of the differential equation of thermal conductivity, for example, by the method of sources [6] for the "shell-plate" welded joint are not acceptable, due to the fact that there is no one-dimensional distribution of the temperature field and the features of welding sources are not taken into account. Therefore, the study of temperature fields in the contour connection is proposed to be carried out by the graphoanalytic method. 
It should be noted that the thermal process is characterized by an unsteady temperature field. There is no axisymmetric loading of the cylinder. The acting non-uniform temperature field does not lead to twisting of the cylindrical shell.

Figure 2 shows the graphical distribution of temperature fields in the radial direction according to the results of the experiment. The experimental data are given in Table 1.
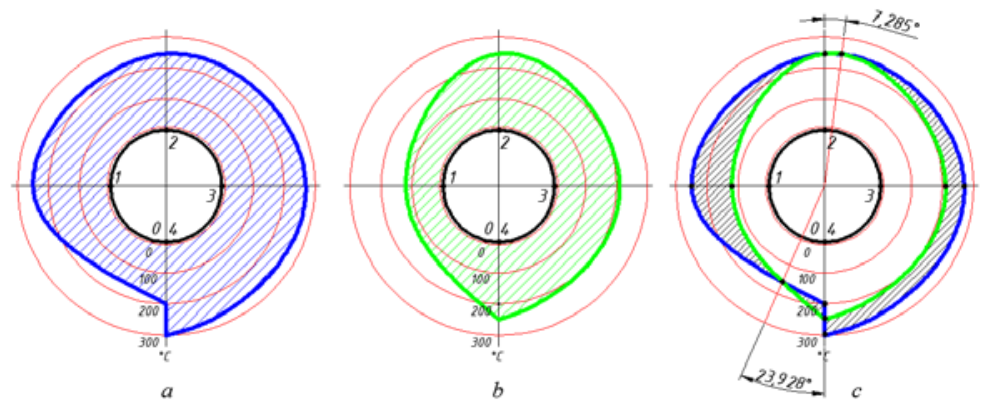

Fig. 2. Distribution of temperature fields in the radial direction of the contour.

Fig. 2, a shows the distribution of the temperature field in the contour of the "shell plate" welded joint two minutes after applying the roller along the entire contour in accordance with the scheme of Fig. 1. There is a relative uniform temperature redistribution from point " 0 "to point" 4». After 7 minutes after applying the roller over the entire contour, the distribution pattern changed, Fig. 2, b. The greatest heat capacity is observed in the sector of the contour "2-4".

The distribution of the temperature field in the contour of the welded joint for the time $\Delta t=300 \mathrm{~s}=5 \mathrm{~min}$ is shown in Fig. 2, c. The highest rate of temperature redistribution is observed in the vicinity of point "1", and in the sector of the contour " $0-1$ " at the intersection of the two curves, the temperature is redistributed to its increase in the coordinate $\varphi=23.928^{\circ} \mathrm{C}$.

In accordance with the theory of welding deformations and stresses $[5,9,10]$, it should be assumed that the vicinity of the near-weld zone $0-1-2$ is most susceptible to the formation of hot cracks.

Figure 3 shows a graph of the rate of temperature change along the contour of the "shell - plate" welded joint at the specified welding modes.

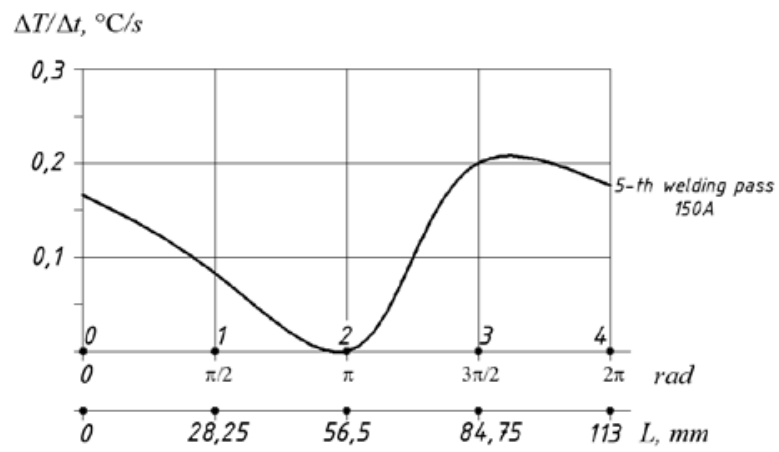

Fig. 3. Graph of the rate of temperature change along the contour of the "shell - plate" welded joint».

On the abscissa axis, the length of the arc and the circumference of the cylinder in radians and millimeters are plotted, and the temperature measurement points are indicated. 
The rate of temperature change, which was determined by the formula (1), is plotted along the ordinate axis.

The curve of the dependence of the rate of temperature change is constructed according to the results of the experiment, Table 1, using the approximation of the linear function by a cubic spline. This approach was used in [7] to obtain an approximate solution of the differential heat equation based on the approximating solution [8] obtained by the graphoanalytic method in the form of constructing a cubic spline passing through the specified points of a simplified function represented graphically by the results of the experiment.

\section{Conclusions}

The proposed graphoanalytic method for determining the temperature fields caused by the action of a highly concentrated heat source makes it possible, based on the theory of welding deformations and stresses, to determine the temperature stresses and deformations both in the joint elements and in the weld contour, and to qualitatively and quantitatively assess the effect of inhomogeneity of the geometric parameters of the welded joint elements on the thermally stressed state of the structure. Minimization of the residual stress state of the welded structure can be carried out by an optimal choice of welding modes.

\section{References}

1. N. Rykalin, Calculation of thermal processes during welding (GLTIMN, Moskow, 1951)

2. L. Mironova, I. Fedik, J. of Machinery Manufacture and Reliability 44 (8) pp. 720-725 (2015)

3. N. Mel'nikov, Design forms and methods of calculation of nuclear reactors (Atomizdat Publ., Moskow, 1972)

4. R. Nigay, E. Nigay, L. Mironova, Investigation of thermal and deformation processes in the welding of shell structures made of carbon and high-alloy structural steels. J. of Physics: Conference Series, 1, p. 1431 (2020)

5. K. Gatovskij, V. Karhin, Theory of welding strains and stresses (LKI, Leningrad, 1980)

6. H. Carslaw, J. Jaeger, Conduction of Heat in Solids (At The Clarendon Press, Oxford, 1959)

7. L. Mironova, R. Nigay, E. Nigay. About one method of modeling high-gradient temperature fields in the welding of shell structures made of carbon and high-alloy structural steels, IOP Conference Series: Materials Science and Engineering, 971(3), 032064 ( 2020)

8. J. Ahlberg, E. Nilson, J. Walsh, The theory of splines and their applications (Academic Press, New York and London, 1967)

9. G. Nikolaev, S. Kurkin, V. Vinokurov, Welded construction. Strength of welded joints and deformation of structures (Vysshaya shkola, Moscow, 1982)

10. V. Vinokurov, Welding deformations and stresses, (Mashinostroenie, Moscow, 1968) 